\title{
Topology design of two-fluid heat exchange
}

\author{
Hiroki Kobayashi $^{1} \cdot$ Kentaro Yaji $^{1}$ (D . Shintaro Yamasaki ${ }^{1} \cdot$ Kikuo Fujita $^{1}$
}

Received: 4 May 2020 / Revised: 24 July 2020 / Accepted: 4 September 2020 / Published online: 2 October 2020

(c) The Author(s) 2020

\begin{abstract}
Heat exchangers are devices that typically transfer heat between two fluids. The performance of a heat exchanger such as heat transfer rate and pressure loss strongly depends on the flow regime in the heat transfer system. In this paper, we present a density-based topology optimization method for a two-fluid heat exchange system, which achieves a maximum heat transfer rate under fixed pressure loss. We propose a representation model accounting for three states, i.e., two fluids and a solid wall between the two fluids, by using a single design variable field. The key aspect of the proposed model is that mixing of the two fluids can be essentially prevented. This is because the solid constantly exists between the two fluids due to the use of the single design variable field. We demonstrate the effectiveness of the proposed method through three-dimensional numerical examples in which an optimized design is compared with a simple reference design, and the effects of design conditions (i.e., Reynolds number, Prandtl number, design domain size, and flow arrangements) are investigated.
\end{abstract}

Keywords Topology optimization · Two kinds of fluids · Heat exchange $\cdot$ Interpolation scheme

\section{Introduction}

Heat exchangers are devices that transfer heat between two or more fluids. Recently, designing high-performance heat exchangers has been prioritized owing to an increased demand for such systems with low energy consumption. Most heat exchangers involve two-fluid heat exchange with indirect contact; i.e., there are two fluids separated by a wall. In such heat exchangers, the performances depend on the flow regime. For instance, the curving and branching of the flow channels of each fluid and the adjacency of the flow channels to each other have a significant effect on the heat transfer rate and the pressure loss. Therefore, consideration of such characteristics is significant in heat exchanger design.

Generally, in heat exchanger design, a type is first selected from the existing ones, and the details, e.g., size, shape,

Responsible Editor: Hyunsun Alicia Kim

Electronic supplementary material The online version of this article (https://doi.org/10.1007/s00158-020-02736-8) contains supplementary material, which is available to authorized users.

Kentaro Yaji

yaji@mech.eng.osaka-u.ac.jp

1 Department of Mechanical Engineering, Graduate School of Engineering, Osaka University, 2-1, Yamadaoka, Suita, Osaka 565-0871, Japan and fin type, are determined (Shah and Sekulic 2003). Optimization methods for the existing types of heat exchangers have been well researched in previous works. For instance, Hilbert et al. (2006) studied a multiobjective optimization method concerning the blade shape of a tubular heat exchanger via a genetic algorithm. Kanaris et al. (2009) applied a response surface method to optimization problems for a plate heat exchanger with undulated surfaces. Guo et al. (2018) proposed a design method to simultaneously determine the fin types and their optimal sizes in plate fin heat exchangers.

The existing types of heat exchangers are typically manufactured through traditional processes such as pressing of plates and bending of tubes. This implies that the typical heat exchangers are designed under strict constraints pertaining to their manufacturability. However, there is an increasing demand to produce heat exchangers more efficient and compact, as the traditional manufacturing techniques are usually too inefficient to satisfy this demand. To produce innovative heat exchangers, these constraints pertaining to their manufacturability should be eliminated. In this regard, using additive manufacturing is a promising option, and has been employed for manufacturing innovative heat exchangers (e.g., Scheithauer et al. 2018) in instances where traditional techniques cannot be easily be applied. According to such progression of additive manufacturing technologies, revealing and understanding the ideal flow regime of the two fluids in heat exchange 
systems become increasingly important. However, the ideal flow regime is not obvious in most cases. For such a problem, topology optimization is a promising methodology, as it enables shape and topological changes of the structure.

Topology optimization was first proposed by Bendsøe and Kikuchi (1988). It essentially comprises introduction of a fixed design domain and replacement of the original optimization problem with a material distribution problem. A characteristic function is introduced to represent either solid material or void at the appropriate points in the design domain. Although topology optimization was originally applied to solid mechanics problems, it has been developed for application in various physical problems (Bendsøe and Sigmund 2003; Sigmund and Maute 2013; Deaton and Grandhi 2014).

For fluid flow problems, Borrvall and Petersson (2003) proposed a topology optimization method for solid/fluid distribution problems in Stokes flow. The critical feature in their method is the introduction of a fictitious body force term, which can discriminate fluid and solid at points in the design domain, according to each design variable value. Their method was extended for NavierStokes equations (Gersborg-Hansen et al. 2005; Olesen et al. 2006), and design problems of fluidic devices, such as microreactors (Okkels and Bruus 2007), micromixers (Andreasen et al. 2009; Deng et al. 2018), Tesla valves (Lin et al. 2015; Sato et al. 2017), and flow batteries (Yaji et al. 2018b; Chen et al. 2019). The thermal-fluid problems that have been studied include forced convection problems (Matsumori et al. 2013; Koga et al. 2013; Yaji et al. 2015), natural convection problems (Alexandersen et al. 2014; Coffin and Maute 2016), and large-scale three-dimensional problems involving cluster computing (Alexandersen et al. 2016; Yaji et al. 2018a). In addition, Dilgen et al. (2018) applied density-based topology optimization to turbulent heat transfer problems. For practical approaches in utilizing topology optimization in complex fluid problems, simplified models have recently been studied for solving turbulent heat transfer problems (Zhao et al. 2018; Yaji et al. 2020; Kambampati and Kim 2020), natural convection problems (Asmussen et al. 2019; Pollini et al. 2020), and heat exchanger design problems (Haertel and Nellis 2017; Kobayashi et al. 2019). The history and the state-of-the-art of topology optimization for fluid problems including heat transfer problems are summarized in the recently published review paper (Alexandersen and Andreasen 2020).

Many studies regarding topology optimization of thermal-fluid problems dealt with solid/fluid distribution; i.e., only one fluid is considered. A topology optimization method for two-fluid and one-solid distribution problems is essential to generate an innovative design of a two-fluid heat exchanger. Papazoglou (2015) first explored topology optimization for designing two-fluid heat exchangers. The results indicated that a multi-material model, which uses two types of design variable fields, is more effective than a tracking model, which uses an advection equation for identifying two fluids. However, the utility of the results is limited by the assumption of a zero thickness wall, which provides an unrealistic situation. Saviers et al. (2019) reported numerical and empirical investigations on a topology-optimized heat exchanger. However, the detailed formulation was withheld as proprietary information. Based on a multi-material model, Tawk et al. (2019) proposed a topology optimization method for twodimensional heat transfer problems involving two-fluid and one-solid domains. In their method, a penalty function is used to prevent the mixing of the two fluids by introducing a non-zero thickness wall. The authors, however, stated that the solution search becomes unstable in certain conditions because of the penalty scheme.

In the general framework of multi-material topology optimization, three states (two types of materials and a void) are accounted for by using multiple design variable fields. Conversely, in topology optimization of two-fluid heat exchange, the two fluids should be separated by a non-zero thickness wall. For two-fluid problems, solving the multimaterial topology optimization typically requires a penalty scheme as with the previous work (Tawk et al. 2019), since the representation model for the multiple states has an excessive degree of freedom. There arises a possibility that a simpler representation model can be obtained by focusing on the characteristics of the two-fluid problems. Haertel (2018) proposed a two-dimensional representation model for designing cross-flow heat exchangers, in which three states — two fluids and a wall_-are represented using a single design variable field with the design field gradient (Clausen et al. 2015).

In this study, we propose a simple representation model for topology optimization of two-fluid heat exchange. We introduce two types of fictitious force terms, one in each flow field, for representing a wall between the two types of fluid, which essentially cannot mix. This idea is related to the previous work of Papazoglou (2015). However, our model can handle a non-zero thickness wall by using a single design variable field.

We emphasize that the two-fluid heat exchange problems should be treated as three-dimensional problems since the topological change is significantly restricted in the case of two-dimensional problems. Thus, we apply the proposed method to three-dimensional heat exchange problems and examine the effects of several design conditions, such as Reynolds number, Prandtl number, design domain size, and flow arrangements. 
The remaining sections of this paper are organized as follows. In Section 2, the design problem of two-fluid heat exchange is presented, as well as the method to represent two fluids and one solid by the single design variable field is briefly discussed. In Section 3, the analysis model and the proposed representation model are presented, and the topology optimization problem is formulated. Furthermore, the numerical implementation is presented. In Section 4, several three-dimensional numerical examples ${ }^{1}$ are provided to validate the proposed method and to discuss the ideal flow regime of the two-fluid heat exchange. Finally, Section 5 concludes this study.

\section{Topology optimization for two-fluid heat exchange}

\subsection{Design problem}

A simple heat exchanger, in which flow channels of the two fluids are separated by a wall as presented in Fig. 1, is considered. An inlet and an outlet of each fluid are respectively set, and each fluid flows independently. The heat is transferred between the two fluids in the design domain $D$. The hot and cold fluids are denoted by "fluid 1 " and "fluid 2," respectively.

In this study, we consider a topology optimization problem for designing the shape and topology of the flow channels and the separating wall in the design domain $D$. The objectives of the heat exchanger design are the maximization of the heat transfer rate and minimization of the pressure loss. This multi-objective design problem is transformed into a single-objective design problem by fixing the pressure loss. The detailed formulation is provided in Section 3.

\subsection{Basic concept of topology optimization}

Topology optimization essentially involves the replacement of the original structural optimization problem with a material distribution problem in a fixed design domain $D$ that contains the original design domain $\Omega$. Bendsøe and Kikuchi (1988) introduced a characteristic function $\chi_{\Omega}$ that assumes a discrete value 0 and 1 , as follows:

$\chi_{\Omega}(\mathbf{x})=\left\{\begin{array}{lll}1 & \text { if } & \mathbf{x} \in \Omega \\ 0 & \text { if } & \mathbf{x} \in D \backslash \Omega,\end{array}\right.$

where $\mathbf{x}$ represents the position in the fixed design domain $D$. Since the characteristic function $\chi_{\Omega}$ is a discontinuous

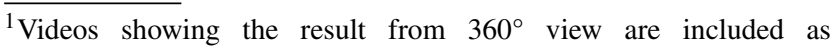
supplementary material.

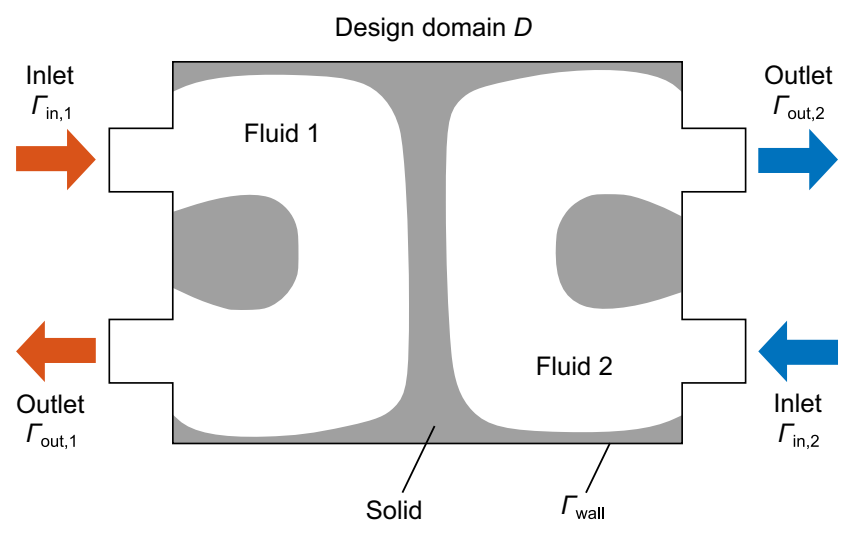

Fig. 1 Schematic diagram of two-fluid heat exchange

function, a relaxation technique is required for gradientbased optimization. In the density-based method (Bendsøe and Sigmund 2003), the characteristic function $\chi_{\Omega}$ is replaced with a continuous scalar function $0 \leq \gamma(\mathbf{x}) \leq 1$, which corresponds to the raw design variable field in the topology optimization problem.

To ensure the spatial smoothness of the material distribution in $D$, the filtering technique is generally applied in topology optimization. In this study, Helmholtz-type filter (Kawamoto et al. 2011; Lazarov and Sigmund 2011) is employed, as follows:

$-R^{2} \nabla^{2} \tilde{\gamma}+\tilde{\gamma}=\gamma$,

where $\tilde{\gamma}$ is a smoothed design variable field, and $R$ is a filtering radius. By solving (2), $\gamma$ is mapped to $\tilde{\gamma}$, thus ensuring a spatial smoothness in $D$.

To elucidate the physical phenomena more accurately, intermediate values of the design variable field need to be eliminated. Therefore, a projection function is used as follows (Wang et al. 2011):

$\hat{\tilde{\gamma}}=\frac{\tanh (\beta \eta)+\tanh (\beta(\tilde{\gamma}-\eta))}{\tanh (\beta \eta)+\tanh (\beta(1-\eta))}$,

where $\hat{\tilde{\gamma}}$ is the design variable field after the projection, and $\beta$ and $\eta$ are the tuning parameters that control the function shape.

\subsection{Representation model}

For the topology optimization of two-fluid heat exchange, the design variable field should represent the two fluids and

Table 1 Representation model of two fluids and one solid in the proposed method

Design variable field

$\gamma=0 \quad 0<\gamma<1 \quad \gamma=1$

Fluid 2

Solid

Fluid 1 
the one solid, as shown in Fig. 1. Here, we discuss the method to represent the two fluids and the one solid by the design variable field.

Table 1 shows the state represented by the design variable field. The design variable field $\gamma$ assumes 1 in fluid 1 and 0 in fluid 2 . The two fluids can consistently be separated by the solid when the spatial smoothness of the design variable field is preserved in the design domain $D$. It should be noted that a filtering technique (2) is essential in the proposed representation model. In addition, as the solid domain is defined as the intermediate value of the design variable field, a projection function (3) is used to represent a thin wall precisely.

The method of incorporating $\hat{\tilde{\gamma}}$ into the governing equations for a two-fluid heat exchange system is discussed in Section 3.1, and the numerical verification of the proposed model is provided in Section 4.2.

\section{Formulation}

\subsection{Governing equations and boundary conditions}

The governing equations of an incompressible steady flow are given as follows:

$\nabla \cdot \mathbf{u}=0$

$\rho(\mathbf{u} \cdot \nabla) \mathbf{u}=-\nabla p+\mu \nabla^{2} \mathbf{u}+\mathbf{F}$,

where $\mathbf{u}$ is the velocity, $\rho$ is the density, $p$ is the pressure, $\mu$ is the viscosity, and $\mathbf{F}$ is a body force. In the fluid topology optimization, $\mathbf{F}$ is a fictitious body force caused by the solid objects in the flow (Borrvall and Petersson 2003). The energy equation without the heat source is defined as follows:

$\rho C_{p}(\mathbf{u} \cdot \nabla) T-k \nabla^{2} T=0$,

where $C_{p}$ is the specific heat at constant pressure, $T$ is the temperature, and $k$ is the thermal conductivity.

The boundary conditions are defined as follows:

$$
\begin{array}{rrrl}
p=p_{\text {in }, 1}, & T=T_{\text {in }, 1} & \text { on } & \Gamma_{\text {in }, 1}, \\
p=p_{\text {out }, 1}, & \nabla T \cdot \mathbf{n}=0 & \text { on } & \Gamma_{\text {out }, 1}, \\
p=p_{\text {in }, 2}, & T=T_{\text {in }, 2} & \text { on } & \Gamma_{\text {in }, 2}, \\
p=p_{\text {out }, 2}, & \nabla T \cdot \mathbf{n}=0 & \text { on } & \Gamma_{\text {out }, 2}, \\
\mathbf{u}=\mathbf{0}, & \nabla T \cdot \mathbf{n}=0 & \text { on } & \Gamma_{\text {wall }},
\end{array}
$$

where subscripts 1 and 2 represent the fluid 1 and fluid 2, respectively. As mentioned in Section 2.1, the constant pressure loss is assumed by fixing the pressure at the inlet and outlet.

\subsection{Proposed interpolation scheme}

In this study, the different fictitious forces in the governing equations for fluid 1 and fluid 2 are imposed separately to realize the state represented by the design variable field indicated in Table 1. Therefore, the governing equations are separated for fluid 1 and fluid 2, as follows:

$\nabla \cdot \mathbf{u}_{1}=0$,

$\rho_{1}\left(\mathbf{u}_{1} \cdot \nabla\right) \mathbf{u}_{1}=-\nabla p_{1}+\mu_{1} \nabla^{2} \mathbf{u}_{1}+\mathbf{F}_{1}$,

$\nabla \cdot \mathbf{u}_{2}=0$

$\rho_{2}\left(\mathbf{u}_{2} \cdot \nabla\right) \mathbf{u}_{2}=-\nabla p_{2}+\mu_{2} \nabla^{2} \mathbf{u}_{2}+\mathbf{F}_{2}$.

The last terms in (13) and (15) are crucial in the proposed method. These fictitious body forces are defined as follows:

$\mathbf{F}_{1}=-\alpha_{1} \mathbf{u}_{1}$,

$\mathbf{F}_{2}=-\alpha_{2} \mathbf{u}_{2}$,

where $\alpha_{1}(\mathbf{x})$ and $\alpha_{2}(\mathbf{x})$ are the inverse permeability of the porous media at position $\mathbf{x}$ (Borrvall and Petersson 2003). The fluid hardly flows when $\alpha_{1}$ or $\alpha_{2}$ assumes a large value. Consequently, the fluid can flow freely when $\alpha_{1}$ or $\alpha_{2}$ assumes a zero value. The inverse permeabilities $\alpha_{1}$ and $\alpha_{2}$ are defined as follows:

$\alpha_{1}(\hat{\tilde{\gamma}})=\alpha_{\max } \frac{q(1-\hat{\tilde{\gamma}})}{q+\hat{\tilde{\gamma}}}$,

$\alpha_{2}(\hat{\tilde{\gamma}})=\alpha_{\max } \frac{q \hat{\tilde{\gamma}}}{q+1-\hat{\tilde{\gamma}}}$

where $\alpha_{\max }$ is the maximum inverse permeability and $q$ is the parameter that controls the convexity of $\alpha$. Both inverse permeabilities are defined based on the interpolation function by Borrvall and Petersson (2003). However, these two interpolation functions are symmetrical with respect to $\hat{\tilde{\gamma}}$. Figure 2 displays the graph of the interpolation functions at $\alpha_{\max }=10^{4}$ and $q=0.01$ in (18) and (19), and $\beta=8$ and $\eta=0.5$ in (3). As revealed in Fig. 2, if $\hat{\tilde{\gamma}}=0, \alpha_{1}$ assumes a large value $\alpha_{\max }$ and $\alpha_{2}$ assumes a zero value. For this reason, only fluid 2 can flow whereas fluid 1 cannot flow. Conversely, if $\hat{\tilde{\gamma}}=1$, only fluid 1 can flow since $\alpha_{1}$ assumes a zero value and $\alpha_{2}$ assumes $\alpha_{\max }$. In the intermediate value of $\hat{\tilde{\gamma}}$, both fluids hardly flow because both $\alpha_{1}$ and $\alpha_{2}$ are not zero. Therefore, the flow characteristics of Table 1 can be achieved via these interpolation functions of the inverse permeability.

To simplify the numerical implementation, the governing equations are replaced with dimensionless form, and the 


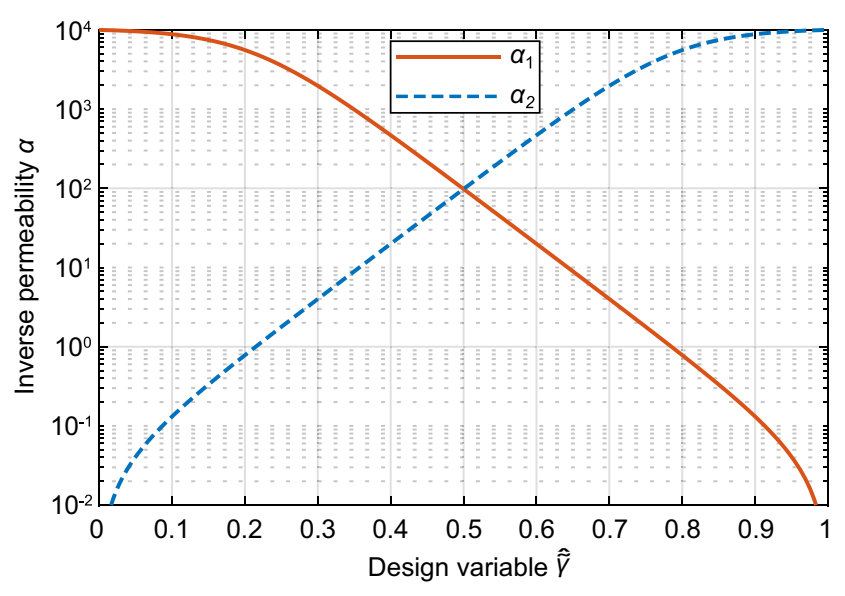

Fig. 2 Interpolation functions for fluid 1 and fluid 2 at $\alpha_{\max }=10^{4}$ and $q=0.01$ in (18) and (19), and $\beta=8$ and $\eta=0.5$ in (3)

dimensionless quantities, i.e., the Reynolds number and the Péclet number, are introduced.

The dimensionless equations of (12)-(15) are defined as follows:

$\nabla^{*} \cdot \mathbf{u}_{1}^{*}=0$

$\left(\mathbf{u}_{1}^{*} \cdot \nabla^{*}\right) \mathbf{u}_{1}^{*}=-\nabla^{*} p_{1}^{*}+\frac{1}{R e_{1}} \nabla^{* 2} \mathbf{u}_{1}^{*}-\alpha_{1}^{*} \mathbf{u}_{1}^{*}$,

$\nabla^{*} \cdot \mathbf{u}_{2}^{*}=0$

$\left(\mathbf{u}_{2}^{*} \cdot \nabla^{*}\right) \mathbf{u}_{2}^{*}=-\nabla^{*} p_{2}^{*}+\frac{1}{R e_{2}} \nabla^{* 2} \mathbf{u}_{2}^{*}-\alpha_{2}^{*} \mathbf{u}_{2}^{*}$,

where $\nabla^{*}=L \nabla$. The superscript asterisk represents dimensionless variables, defined by a characteristic length $L$ and characteristic speeds $U_{1}$ and $U_{2}$, as follows:

$\mathbf{u}_{1}^{*}=\frac{\mathbf{u}_{1}}{U_{1}}, \quad p_{1}^{*}=\frac{p_{1}-p_{\text {out }, 1}}{\rho_{1} U_{1}^{2}}$,

$\alpha_{1}^{*}=\frac{L \alpha_{1}}{\rho_{1} U_{1}}, \quad R e_{1}=\frac{\rho_{1} U_{1} L}{\mu_{1}}$,

$\mathbf{u}_{2}^{*}=\frac{\mathbf{u}_{2}}{U_{2}}, \quad p_{2}^{*}=\frac{p_{2}-p_{\text {out }, 2}}{\rho_{2} U_{2}^{2}}$,

$\alpha_{2}^{*}=\frac{L \alpha_{2}}{\rho_{2} U_{2}}, \quad R e_{2}=\frac{\rho_{2} U_{2} L}{\mu_{2}}$.

The characteristic speeds $U_{1}$ and $U_{2}$ cannot be defined using the magnitude of the inlet velocity because the inlet pressure is fixed, as mentioned in Section 3.1. Therefore, $U_{1}$ and $U_{2}$ are defined using the pressure loss (Yaji et al. 2015), as follows:

$$
\begin{aligned}
& U_{1}=\sqrt{\frac{p_{\text {in }, 1}-p_{\text {out }, 1}}{\rho_{1}}}, \\
& U_{2}=\sqrt{\frac{p_{\text {in }, 2-p_{\text {out }, 2}}}{\rho_{2}} .}
\end{aligned}
$$

As a result, the proposed interpolation functions $\alpha_{1}^{*}(\hat{\tilde{\gamma}})$ and $\alpha_{2}^{*}(\hat{\tilde{\gamma}})$ in the dimensionless form are given as:

$\alpha_{1}^{*}(\hat{\tilde{\gamma}})=\alpha_{\max }^{*} \frac{q(1-\hat{\tilde{\gamma}})}{q+\hat{\tilde{\tilde{\gamma}}}}$,
$\alpha_{2}^{*}(\hat{\tilde{\gamma}})=\alpha_{\max }^{*} \frac{q \tilde{\gamma}}{q+1-\hat{\tilde{\gamma}}}$,

where $\alpha_{\max }^{*}$ is the maximum inverse permeability.

The dimensionless equation of (6) is defined as follows:

$\mathbf{u}^{*} \cdot \nabla^{*} T^{*}-\frac{1}{P e} \nabla^{* 2} T^{*}=0$.

Here, $P e$ is the Péclet number $P e=\operatorname{Pr} R e$, in which $P r$ is the Prandtl number given for the three states, i.e., fluid 1, fluid 2, and the solid. The dimensionless temperature $T^{*}$ and these Prandtl numbers, $P r_{1}, P r_{2}$, and $P r_{\mathrm{s}}$, are respectively defined as follows:

$$
\begin{aligned}
T^{*} & =\frac{T-T_{\mathrm{in}, 2}}{T_{\mathrm{in}, 1}-T_{\mathrm{in}, 2}}, \\
\operatorname{Pr}_{1} & =\frac{\mu_{1} C_{p, 1}}{k_{1}}, \quad P r_{2}=\frac{\mu_{2} C_{p, 2}}{k_{2}}, \quad P r_{\mathrm{s}}=\frac{\bar{\mu} C_{p, \mathrm{~s}}}{k_{\mathrm{s}}},
\end{aligned}
$$

where $\bar{\mu}=\left(\mu_{1}+\mu_{2}\right) / 2$, and subscript s represents the solid. In (30), since the dimensional parameters of thermal-fluid properties are integrated into the dimensionless parameter, $P e$, an interpolation function should be introduced so that $P e$ depends on the states, i.e., fluid 1, fluid 2, or the solid. Therefore, we propose an interpolation function based on the Gaussian function, as follows:

$$
\begin{aligned}
P e(\hat{\tilde{\gamma}})= & \left(P e_{\mathrm{s}}-\frac{P e_{1}+P e_{2}}{2}\right) \exp \left(-\frac{(\hat{\tilde{\gamma}}-0.5)^{2}}{2 s^{2}}\right) \\
& +P e_{2}+\left(P e_{1}-P e_{2}\right) \hat{\tilde{\gamma}},
\end{aligned}
$$

where $s$ is the parameter that controls the shape of the Gaussian function. $P e_{1}=P r_{1} R e_{1}, P e_{2}=P r_{1} R e_{1}$, and $P e_{\mathrm{s}}=P r_{\mathrm{s}}\left(R e_{1}+R e_{2}\right) / 2$ are the Péclet numbers of fluid 1 ,

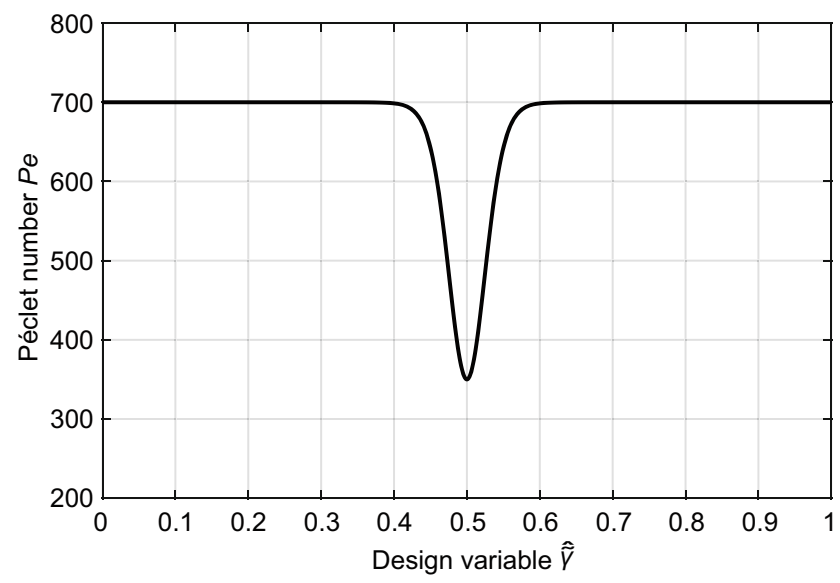

Fig. 3 Interpolation function at $P e_{1}=700, P e_{2}=700, P e_{\mathrm{s}}=350$, and $s=0.1$ in (18) and (19), and $\beta=8$ and $\eta=0.5$ in (3) 
fluid 2, and the solid, respectively. As shown in Fig. 3, Pe in the intermediate region corresponding to the solid can be smaller than that of the two fluids. This indicates that the solid has larger thermal conductivity than that of the two fluids, under the condition that the remaining parameters of thermal-fluid properties are fixed.

\subsection{Optimization formulation}

As discussed in Section 2.1, the objective of the design problem is to maximize the total heat transfer with a fixed pressure loss. The total heat transfer is given as follows:

$Q=\dot{m} C_{p}\left(\bar{T}_{\text {in }}-\bar{T}_{\text {out }}\right)$,

where $\dot{m}$ is the mass flow, and $\bar{T}$ is the mean temperature. Introducing the boundary integral form, the equation for total heat transfer can be rewritten as:

$Q=C_{p} \int_{\Gamma_{\text {out }}} \rho \mathbf{u} \cdot \mathbf{n} d \Gamma\left(\frac{\int_{\Gamma_{\text {in }}}(\mathbf{u} \cdot \mathbf{n}) T d \Gamma}{\int_{\Gamma_{\text {in }}} \mathbf{u} \cdot \mathbf{n} d \Gamma}-\frac{\int_{\Gamma_{\text {out }}}(\mathbf{u} \cdot \mathbf{n}) T d \Gamma}{\int_{\Gamma_{\text {out }}} \mathbf{u} \cdot \mathbf{n} d \Gamma}\right)$.

Since incompressible flow is assumed in this study, the density is constant and the volume flows at the inlet and outlet are equal. In addition, the inlet temperature $T$ is constant as defined in (7) and (9). Thus, the equation can be further rewritten as:

$Q=\rho C_{p} \int_{\Gamma_{\mathrm{out}}}(\mathbf{u} \cdot \mathbf{n})\left(T_{\mathrm{in}}-T\right) d \Gamma$.

To replace the objective function with dimensionless form, a total heat transfer $Q$ is nondimensionalized as follows:

$Q^{*}=\frac{Q}{\rho C_{p} U L^{2}\left(T_{\mathrm{in}, 1}-T_{\mathrm{in}, 2}\right)}$.

Based on (36), we define the objective function in this study as the sum of the total heat transfer in fluid 1 and fluid 2, as follows:

$J=\int_{\Gamma_{\mathrm{out}, 1}}\left(\mathbf{u}^{*} \cdot \mathbf{n}^{*}\right)\left(1-T^{*}\right) d \Gamma+\int_{\Gamma_{\mathrm{out}, 2}}\left(\mathbf{u}^{*} \cdot \mathbf{n}^{*}\right) T^{*} d \Gamma$.

For brevity, the asterisk symbol of the dimensionless variables is dropped henceforth.

Finally, the topology optimization problem is formulated as follows:

$\underset{\gamma(\mathbf{x})}{\operatorname{maximize}} \quad J=\int_{\Gamma_{\text {out }, 1}}(\mathbf{u} \cdot \mathbf{n})(1-T) d \Gamma+\int_{\Gamma_{\text {out }, 2}}(\mathbf{u} \cdot \mathbf{n}) T d \Gamma$,

subject to $0 \leq \gamma(\mathbf{x}) \leq 1 \quad$ for $\forall \mathbf{x} \in D$,

where the thermal-fluid field is governed by (20)-(23), and (30).

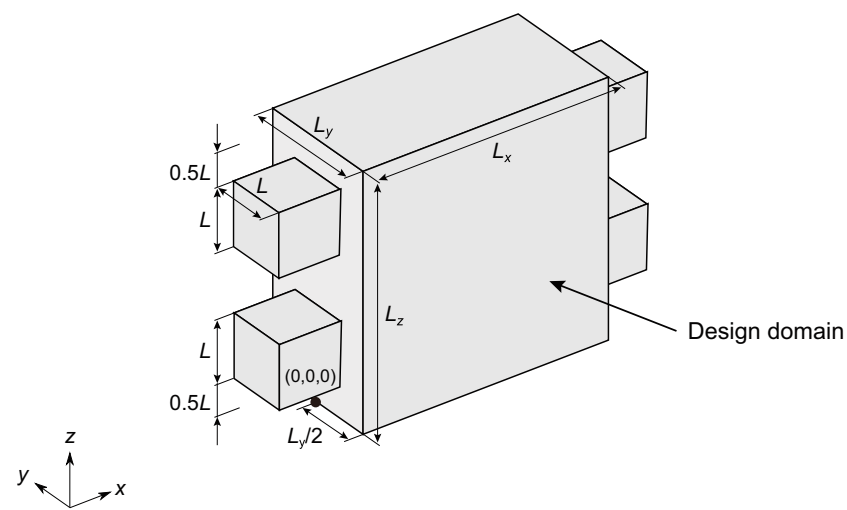

Fig. 4 Analysis domain and its dimensions for two-fluid heat exchange. The rectangular parallelepiped domain is the design domain, and the cubic domains are the non-design domain. $(0,0,0)$ is the coordinate origin

\subsection{Numerical implementation}

The numerical solution of the governing (20)-(23) and (30) is obtained using COMSOL Multiphysics 5.5. The analysis domain is discretized using tetrahedral finite elements. The design variable field $\gamma(\mathbf{x})$ is transformed into a discrete form $\gamma_{i}(i=1, \ldots, n)$, where $n$ is the total number of nodal points in the design domain $D$. The initial values of $\gamma_{i}$ are set to 0.5 uniformly. For the sensitivity analysis, we employ the adjoint method based on the discrete formulation described in the literature (Bendsøe and Sigmund 2003).

The optimization procedure in this study is described as follows:

Step 1. The initial values of $\gamma_{i}$ are set in $D$.

Step 2. The smoothed design variables $\tilde{\gamma}_{i}$ are derived using the Helmholtz-type filter (2), and are projected to $\hat{\tilde{\gamma}}_{i}$ using the projection function (3).

Step 3. The objective function in (38) is calculated by solving the governing (20)-(23), and (30).

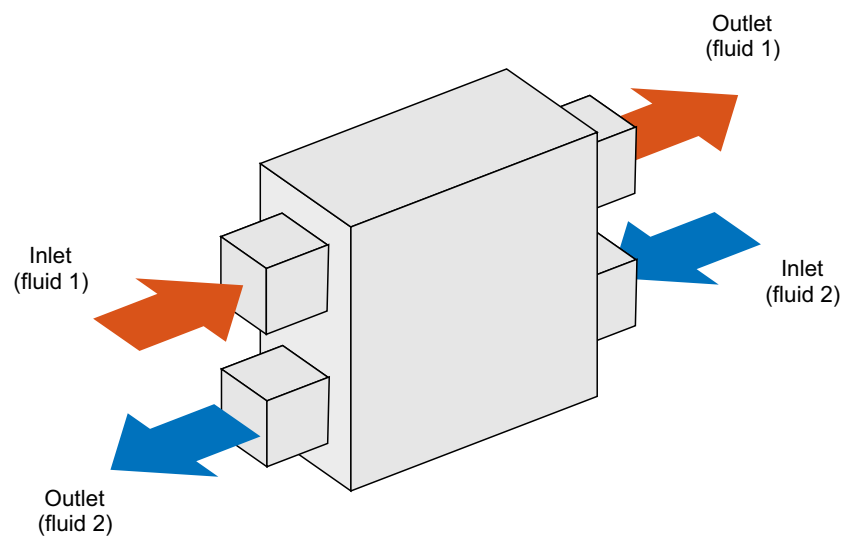

Fig. 5 Schematic diagram of counter flow 


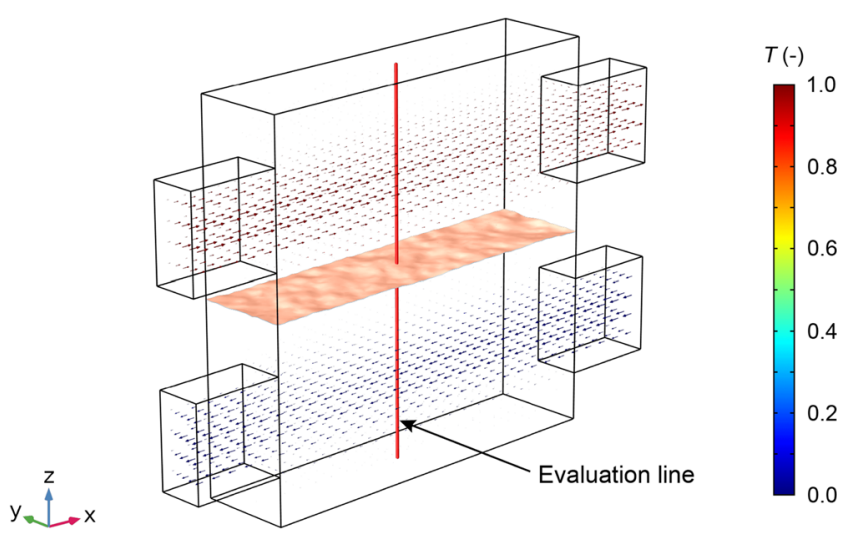

Fig. 6 Simple plate model for verification of the proposed method. The plate is represented as the isosurface of $\hat{\tilde{\gamma}}=0.5$. The evaluation line is located at $\left(L_{x} / 2, L_{y} / 4, z\right)$

Step 4. If the value of $J$ is converged, the optimization process ends. Otherwise, the sensitivity is calculated using the adjoint method.

Step 5. The design variables are updated using sequential linear programming (SLP), and the optimization procedure then returns to Step 2.

\section{Numerical examples}

\subsection{Analysis model}

Figure 4 shows the three-dimensional analysis domain and its dimensions. The dimensions $L_{x}, L_{y}$, and $L_{z}$
Table 2 Mesh dependency in the numerical result of the high-fidelity model in Fig. 7b

\begin{tabular}{lll}
\hline Element number & $J$ & Discrepancy $(\%)$ \\
\hline $9.1 \times 10^{4}$ & 0.0206 & 8.3 \\
$5.2 \times 10^{5}$ & 0.0218 & 2.3 \\
$1.3 \times 10^{6}$ & 0.0223 & - \\
\hline
\end{tabular}

are set to $4 L, 2 L$, and $4 L$, respectively. A symmetrical boundary condition is imposed in the $Z X$ plane. Hence, the computational domain is only half of that shown in Fig. 4, with the symmetrical boundary condition.

The analysis domain is discretized using $5.3 \times 10^{5}$ tetrahedral elements. The inlets and outlets of the two fluids are set as shown in Fig. 5, which outlines the counter flow. The dimensionless quantities are set to $R e_{1}=100$, $R e_{2}=100, P r_{1}=7, P r_{2}=7$, and $P r_{\mathrm{s}}=3.5$. Note that, in this paper, these dimensionless parameter values are not supposed to those of practical heat exchangers.

The parameters regarding the interpolation functions are $\alpha_{\max }=1 \times 10^{4}, q=0.01$, and $s=0.1$. The filtering radius $R$ in (2) is set to $L / 12$ and the parameters for the projection function in (3) are set to $\beta=8$, and $\eta=0.5$, respectively.

\subsection{Verification on a simple plate model}

To verify the applicability of the proposed representation model, we evaluate its accuracy with a simple plate model. As shown in Fig. 6, the plate corresponding to the wall
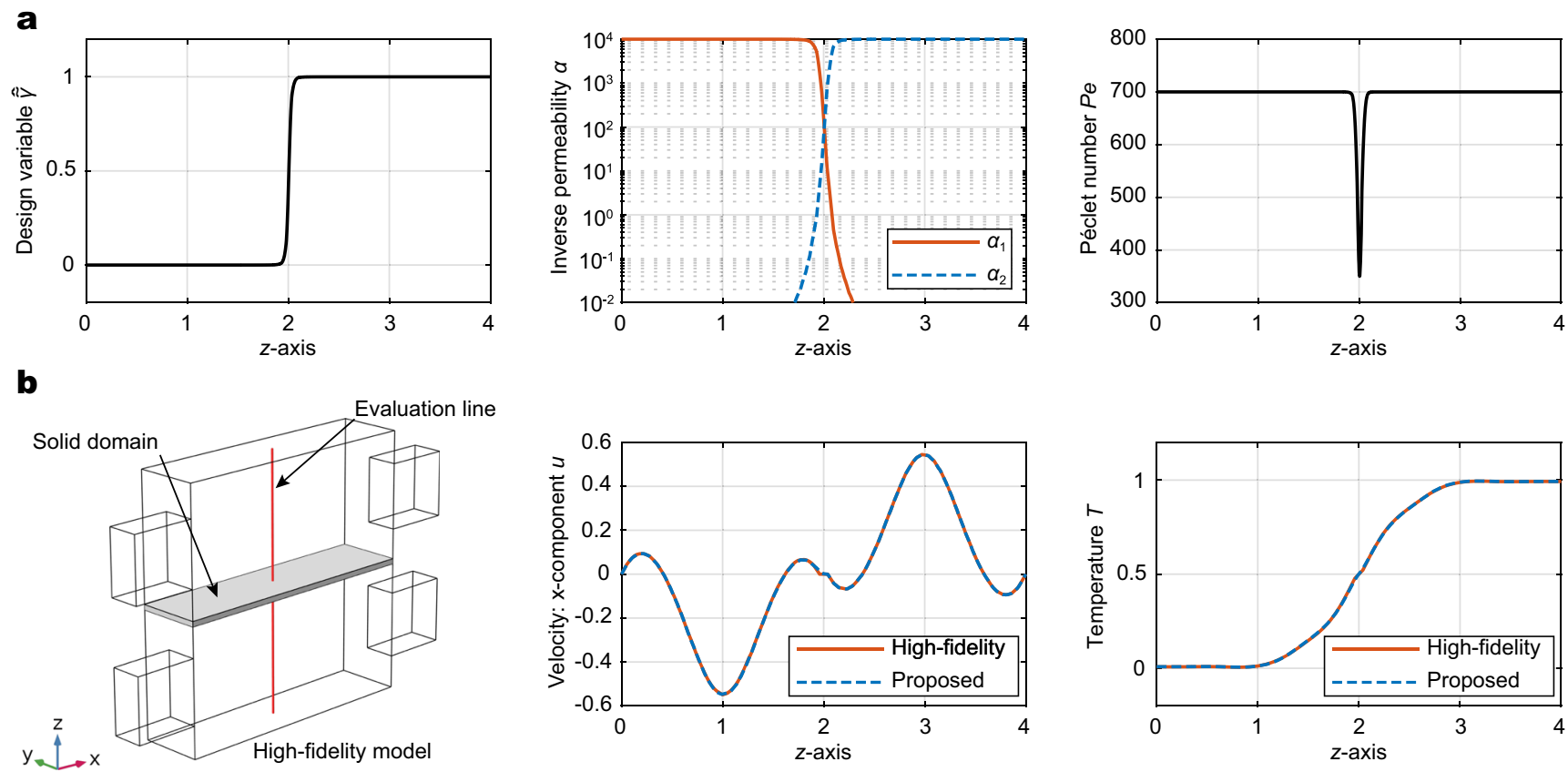

Fig. 7 Numerical results for the verification of the simple plate model: a distribution of $\hat{\tilde{\gamma}}, \alpha$ and $P e$; $\mathbf{b}$ comparison with a high-fidelity model. In the high-fidelity model, a plate, with a thickness of $0.08 L$, is located at $z=L_{z} / 2$. The evaluation line is located at $\left(L_{x} / 2, L_{y} / 4, z\right)$ 


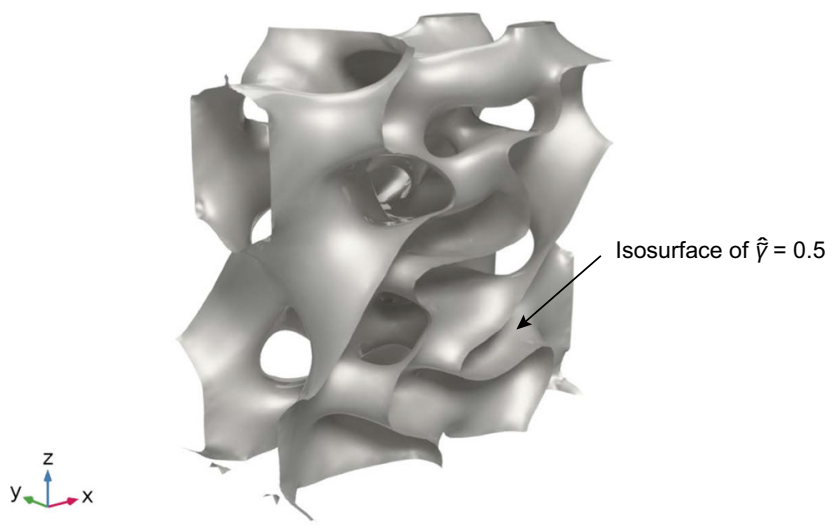

Fig. 8 Isosurface of $\hat{\tilde{\gamma}}=0.5$ of the optimized result. The half domain is optimized with the symmetrical boundary condition on $Z X$ plane

between the two fluids is placed at $z=L_{z} / 2$. To represent the plate, $\gamma=1\left(z \geq L_{z} / 2\right)$ and $\gamma=0\left(z<L_{z} / 2\right)$ are set, respectively. Besides, $\gamma$ is projected to $\hat{\tilde{\gamma}}$ via using the PDE filter and the projection function.

Figure $7 \mathrm{a}$ shows the distributions of $\hat{\tilde{\gamma}}, \alpha$, and $P e$ along the evaluation line shown in Fig. 6, where it can be confirmed that $\hat{\tilde{\gamma}}$ sharply increases from 0 to 1 between $L_{z} / 2 \pm \Delta d / 2$, with $\Delta d \approx 0.08 L$. It should be noted that $\Delta d$ is related to the thickness of the plate, which can be controlled by changing the parameters such as $R$ and $\beta$. However, explicit control of the plate thickness is difficult in the proposed representation model, as many parameters are implicitly dependent upon the thickness. Therefore, the thickness is determined via comparison with a high-fidelity model that has an exact solid plate, as shown in the leftmost panel of Fig. $7 \mathrm{~b}$.

Table 2 shows the objective function $J$ of the highfidelity model and its discrepancy for the three cases of different resolutions. As the discrepancy between the

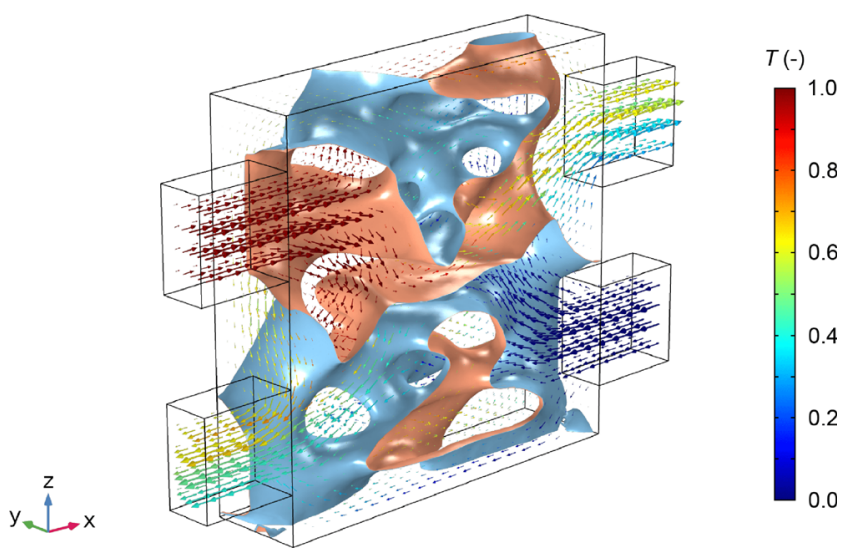

Fig. 9 Optimized result with velocity vectors, where the colors represent the temperature of the fluids. Objective function value: $J=$ 0.321 case of $5.2 \times 10^{5}$ and $1.3 \times 10^{6}$ elements is $2.3 \%$, the analysis domain is discretized using $5.2 \times 10^{5}$ elements. By conducting the preliminary experiments, the thickness of the high-fidelity model is set to $0.08 \mathrm{~L}$. From Fig. $7 \mathrm{~b}$, it can be confirmed that the velocity and the temperature of the proposed representation model are in good agreement with those of the high-fidelity model.

\subsection{Optimized design}

Figure 8 shows the isosurface of $\hat{\tilde{\gamma}}=0.5$. Note that only half of the domain is optimized with the symmetrical boundary condition on $Z X$ plane. Besides, the half sectional view is shown in Fig. 9, where the separating wall is colored with orange on fluid 1 side, and light blue on fluid 2 side. The color of the arrows represents the temperature of the fluids.

The optimization history of $\hat{\tilde{\gamma}}$ is illustrated in Fig. 10, in which fluid 1 and fluid 2 domains appear as the optimization proceeds. In this study, the maximum iteration number of the optimization is set to 100 .

Figure 11 shows the results for the accuracy check as with the case of the simple plate model shown in Fig. 7. The analysis domain of the high-fidelity model is discretized using $5.9 \times 10^{5}$ tetrahedral elements, which are chosen in a similar way to the values in Table 2. The high-fidelity model in the leftmost panel of Fig. 11b is constructed from the result in Fig. 9 so that the thickness of the solid domain is approximately equal to $0.08 \mathrm{~L}$. For this, the solid domain is defined as $0.1 \leq \hat{\tilde{\gamma}} \leq 0.9$. Note that the range of $\hat{\tilde{\gamma}}$ for representing the solid is dependent of the parameters such as $R$ and $\beta$, as discussed in Section 4.2.

From the results in Fig. 11b, the velocity and the temperature along the evaluation line in the optimized design are in good agreement with those of the highfidelity model, while a slight error is observed. Besides, Fig. 12 shows the results of the two-dimensional slice at $y=L_{y} / 4$, with an error is also observed in Fig. 12 b. This discrepancy can be reduced by increasing $\beta$ during the optimization process. However, such a technique typically causes numerical oscillation in terms of the objection function, and is therefore omitted in this study for simplicity. The values of the objective function $J$ in the optimized design and its high-fidelity model are 0.321 and 0.326 , respectively. Hence, the error of the optimized design using the proposed representation model over the high-fidelity model is $1.6 \%$.

As shown in Figs. 8 and 9, geometrically complex flow channels are obtained. The flow channels are gradually curved and branched to circulate the fluids in the whole heat exchange domain, and fluid 1 and fluid 2 are placed alternately. This is because increasing the heat transfer area without the sharp bend is effective for improving the 


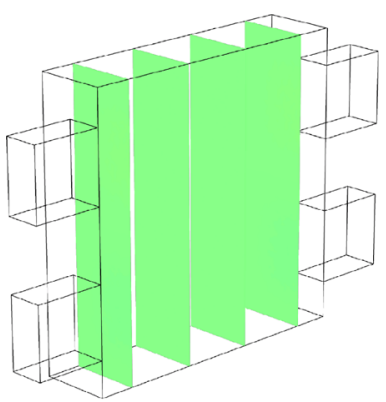

Initial configuration

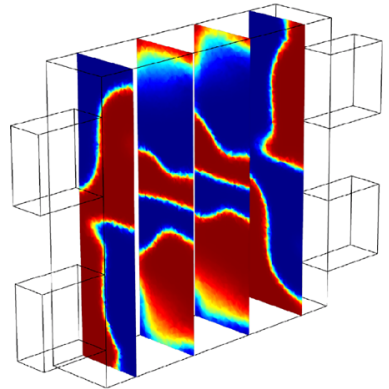

20 step

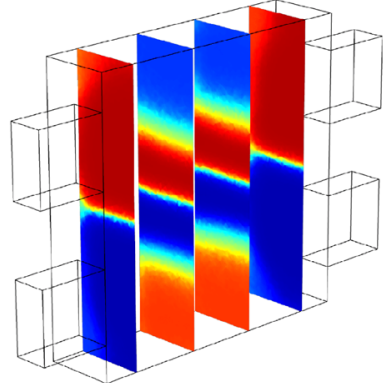

5 step

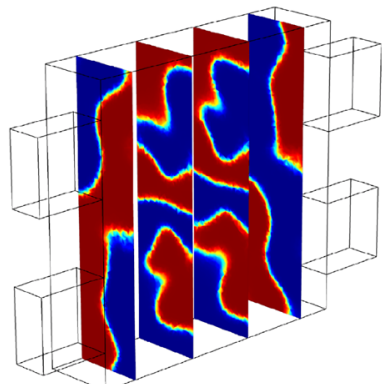

30 step

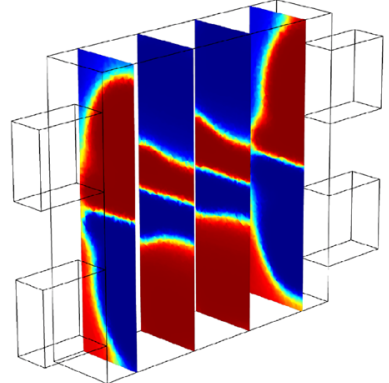

10 step

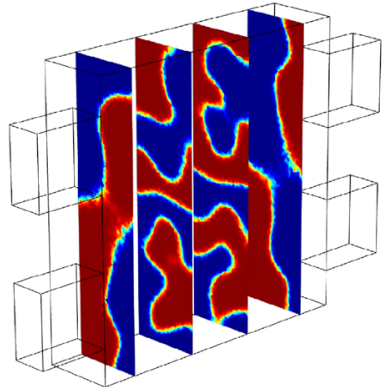

50 step

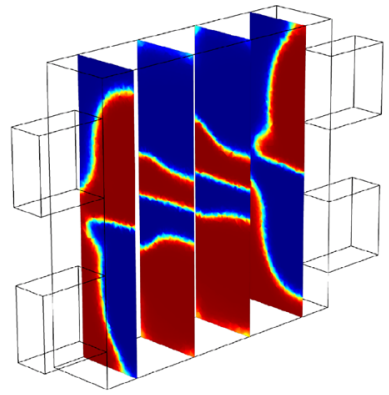

15 step

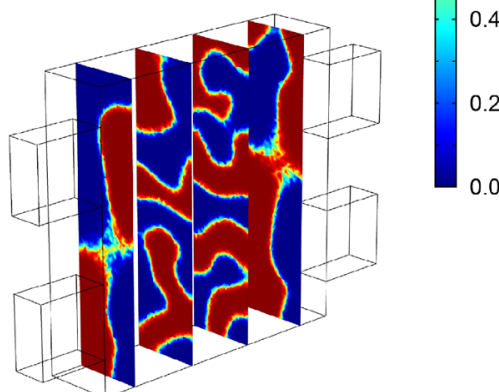

100 step (Optimized design)

$\hat{\tilde{Y}}(-)$

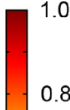

0.4

0.2
0.8

0.6

0.0

Fig. 10 Optimization history of $\hat{\tilde{\gamma}}$ in $Y Z$ slices total heat transfer with low pressure loss. In addition, it is found that the outlet temperature of fluid 1 is lower than that of fluid 2. As illustrated in Fig. 9, the downstream region of fluid 1 adjoins the upstream region of fluid 2 and vice versa. Thus, the fluids can exchange heat in the downstream region, whereas the heat is already transferred in the upstream branched region. This feature is unique to the counter flow and can enhance the heat transfer. a

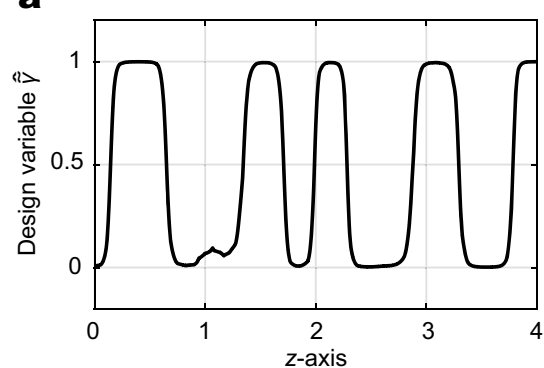

b

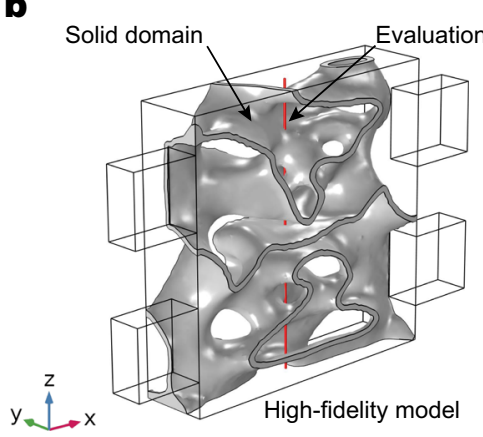

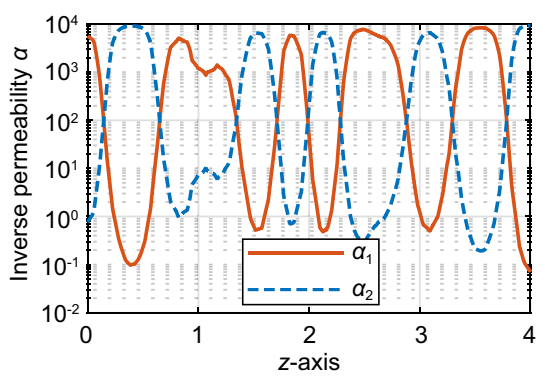

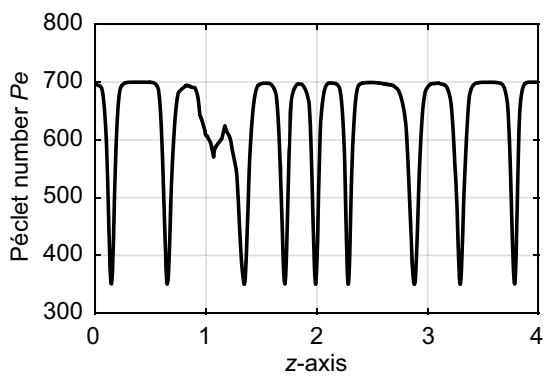

Fig. 11 Numerical results for the verification of the optimized result: a distribution of $\hat{\tilde{\gamma}}, \alpha$ and $P e$; b comparison with a high-fidelity model. In the high-fidelity model, a solid domain is defined as $0.1 \leq$
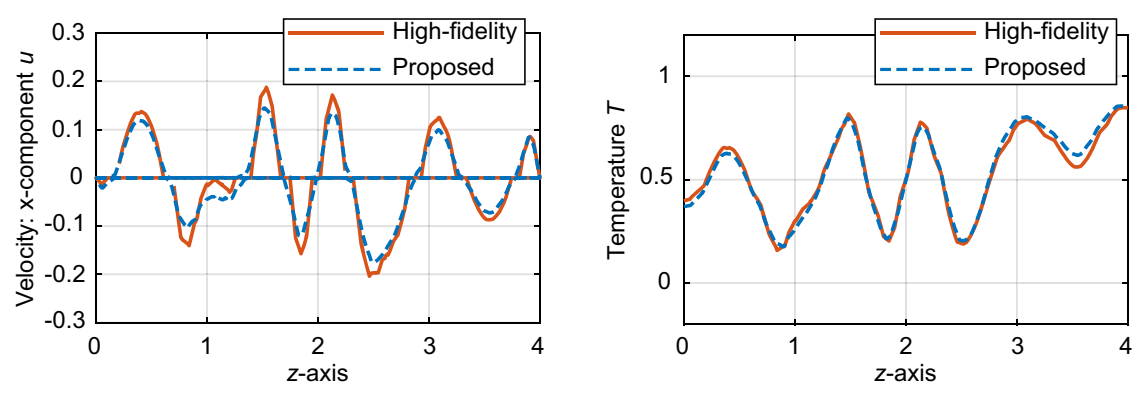

$\hat{\tilde{\gamma}} \leq 0.9$ so that the thickness is approximately equal to $0.08 L$. The evaluation line is located at $\left(L_{x} / 2, L_{y} / 4, z\right)$ 


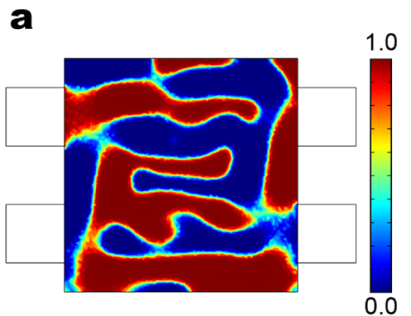

Design variable $\hat{\tilde{Y}}$

b

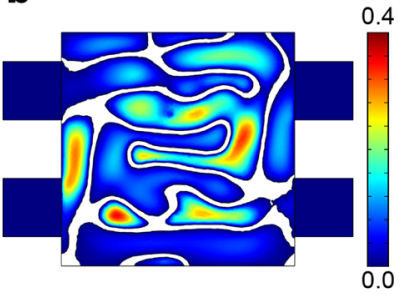

High-fidelity $|\mathbf{u}|$

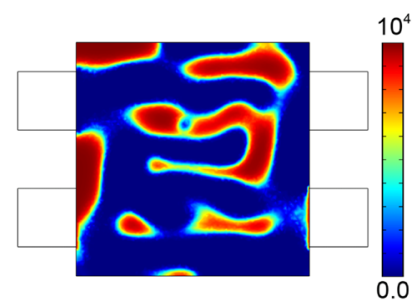

Inverse permeability $\alpha_{1}$

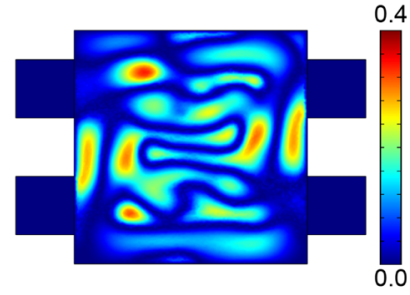

Proposed |u|

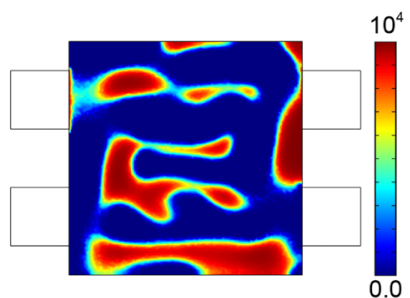

Inverse permeability $\alpha_{2}$

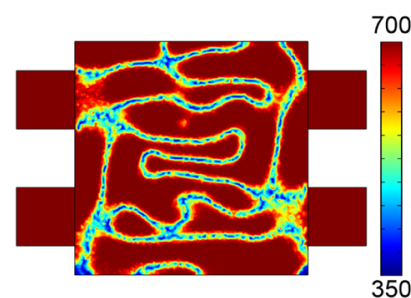

Péclet number $P e$

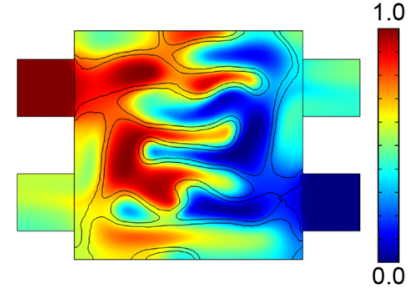

High-fidelity $T$

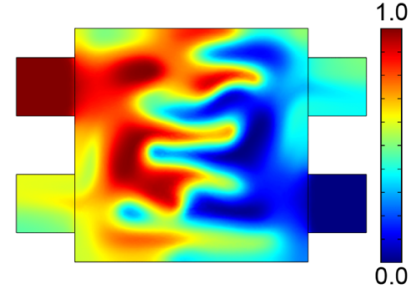

Proposed T

Fig. 12 Numerical results of $Z X$ plane at $y=L_{y} / 4$ for the verification of the optimized result: a distribution of $\hat{\tilde{\gamma}}, \alpha$ and $P e$; b comparison with the high-fidelity model

\subsection{Performance comparison with a reference design}

We now verify the performance of the optimized design, shown in the leftmost panel of Fig. 11b, by comparison with a reference design shown in Fig. 13, which refers to a simple configuration of a multiple plate heat exchanger (Shah and Sekulic 2003). Note that the volume of the solid in the reference model is equal to that of the optimized design.

Table 3 shows the comparison of the total heat transfer $J$, the flow rate of fluid 1 and fluid 2, i.e., $\dot{V}_{1}$ and $\dot{V}_{2}$, and the mean outlet temperature of fluid 1 and fluid 2, i.e., $\bar{T}_{\text {out }, 1}$ and $\bar{T}_{\text {out, } 2 \text {. The flow rate of the reference design }}$ is approximately equal to that of the optimized design. However, there is not a large difference between each mean outlet temperature and each inlet temperature in the reference design. Consequently, the total heat transfer of the optimized design is approximately three times higher

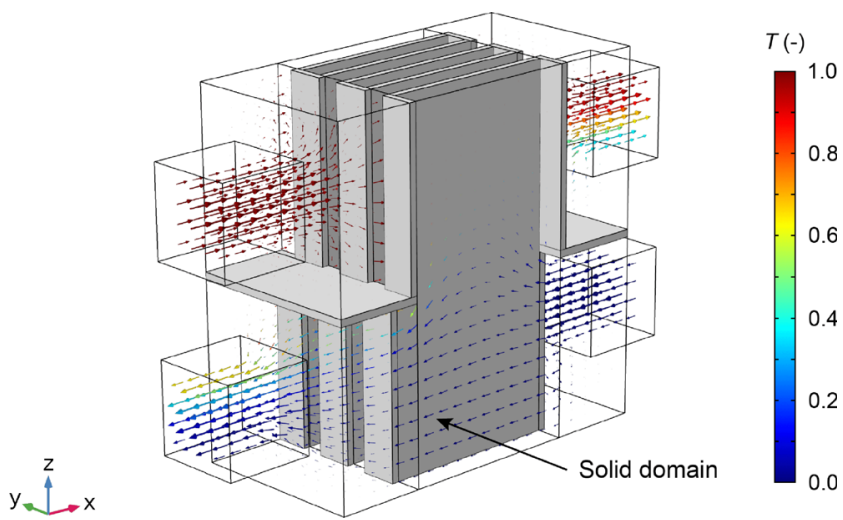

Fig. 13 Reference design. The thickness of the solid domain is $0.08 \mathrm{~L}$. The volume of the solid domain is equal to that of the high-fidelity model of the optimized design in Fig. 11b than that of the reference design. Therefore, it is observed that the optimized design enhances heat transfer under the investigated operating condition.

\subsection{Effects of design conditions}

\subsubsection{Effect of Reynolds number}

Here, we investigate the effect of Reynolds number, $R e$, settings on the optimized configuration. In this study, the Reynolds number represents the pressure loss because the characteristic speed is based on the pressure loss as defined in (26). Figure 14 illustrates the optimized results in the case of $R e_{1}=50, R e_{2}=50$, and the case of $R e_{1}=200$, $R e_{2}=200$. The parameters except for the Reynolds number are identical to the case of $R e_{1}=100, R e_{2}=100$ presented in Section 4.3.

From Figs. 9 and 14, the number of branches increases as the Reynolds number increases. In Fig. 14, a relatively simple structure is obtained in low $R e$. The heat transfer surface is approximately planar instead of the circular channels. Conversely, narrow branched channels are obtained for high $R e$. This is because the heat transfer area can be increased when the high pressure loss is allowed.

Table 3 Thermal fluid performance of the optimized design and reference design, where $J$ is the total heat transfer, $\dot{V}_{1}$ and $\dot{V}_{2}$ are the flow rates of fluid 1 and fluid 2 , and $\bar{T}_{\text {out, } 1}$ and $\bar{T}_{\text {out,2 }}$ are the mean outlet temperature of fluid 1 and fluid 2

\begin{tabular}{llllll}
\hline & $J$ & $\dot{V}_{1}$ & $\dot{V}_{2}$ & $\bar{T}_{\text {out }, 1}$ & $\bar{T}_{\text {out }, 2}$ \\
\hline Optimized & 0.326 & 0.317 & 0.317 & 0.487 & 0.509 \\
Reference & 0.124 & 0.283 & 0.283 & 0.783 & 0.217 \\
\hline
\end{tabular}


a
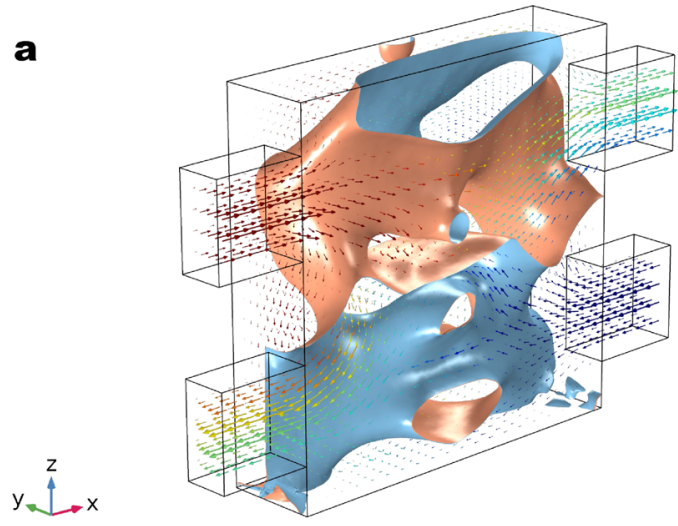

b

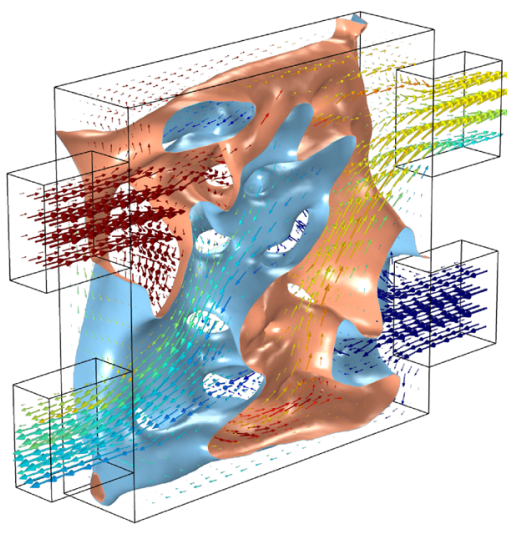

$T(-)$

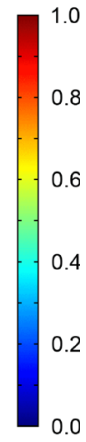

$\mathrm{Z}_{\mathrm{L}}^{\mathrm{L}} \mathrm{x}$
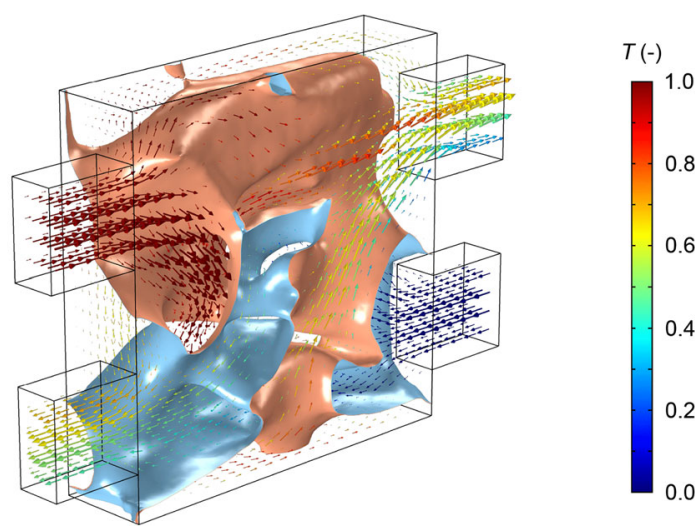

Fig. 15 Optimized result for Reynolds number $R e_{1}=200, R e_{2}=50$. Objective function value: $J=0.310$

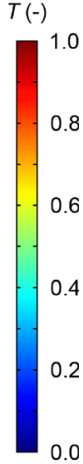

rates of fluid 1 and fluid 2 are $\dot{V}_{1}=0.340$ and $\dot{V}_{2}=$ 0.260 , respectively, which are significantly different from the case using the same $R e$ settings, as shown in Table 3 . Consequently, it is observed that the flow channel of fluid 1 is relatively thinner and more deviant than the flow channel of fluid 2 .

\subsubsection{Effect of Prandtl number}

Figure 16 shows the optimized results in a case of $\operatorname{Pr}_{1}=14$, $P r_{2}=14$. The parameters except for the Prandtl number are similar to the case of $P r_{1}=7, P r_{2}=7$ presented in Section 4.3. The Prandtl number shown in (31) is defined as the ratio of momentum diffusivity to thermal diffusivity. Hence, high Prandtl number results in low total heat transfer.

In Fig. 16, complex flow channels are obtained, which is similar to the case of $R e_{1}=200, R e_{2}=200$ shown in Fig. 14b. The reason for this similarity is related to the trade-off between the total heat transfer and the pressure loss. Increasing the Reynolds number corresponds with the setting performs better than the alternative designs for setting. These results indicate that the ideal topology of the two fluids varies by the Reynolds number setting.

Figure 15 shows the optimized design in which a different Reynolds number, $R e_{1}=200$ and $R e_{2}=50$, is set for each fluid. As the Reynolds number is defined using the pressure loss in this study, a higher Reynolds number can lead to a larger flow rate. Furthermore, the flow

Table 4 Cross-check of $J$ with respect to $R e$

\begin{tabular}{llll}
\hline Analysis $R e$ & \multicolumn{3}{l}{ Optimization Re } \\
& 50 & 100 & 200 \\
\hline 50 & $\mathbf{0 . 2 7 1}$ & 0.263 & 0.244 \\
100 & 0.272 & $\mathbf{0 . 3 2 1}$ & 0.320 \\
200 & 0.215 & 0.289 & $\mathbf{0 . 3 2 9}$ \\
\hline
\end{tabular}

Data in bold highlight the main diagonal components

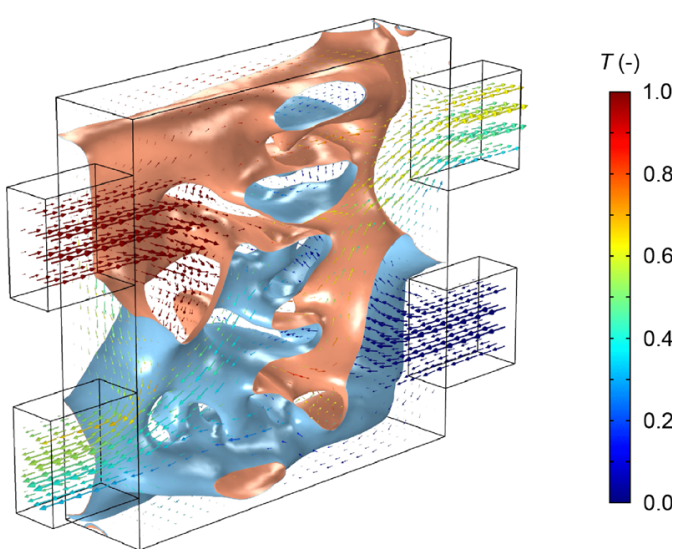

Fig. 16 Optimized result for Prandtl number $P r_{1}=14, P r_{2}=14$. Objective function value: $J=0.265$ 


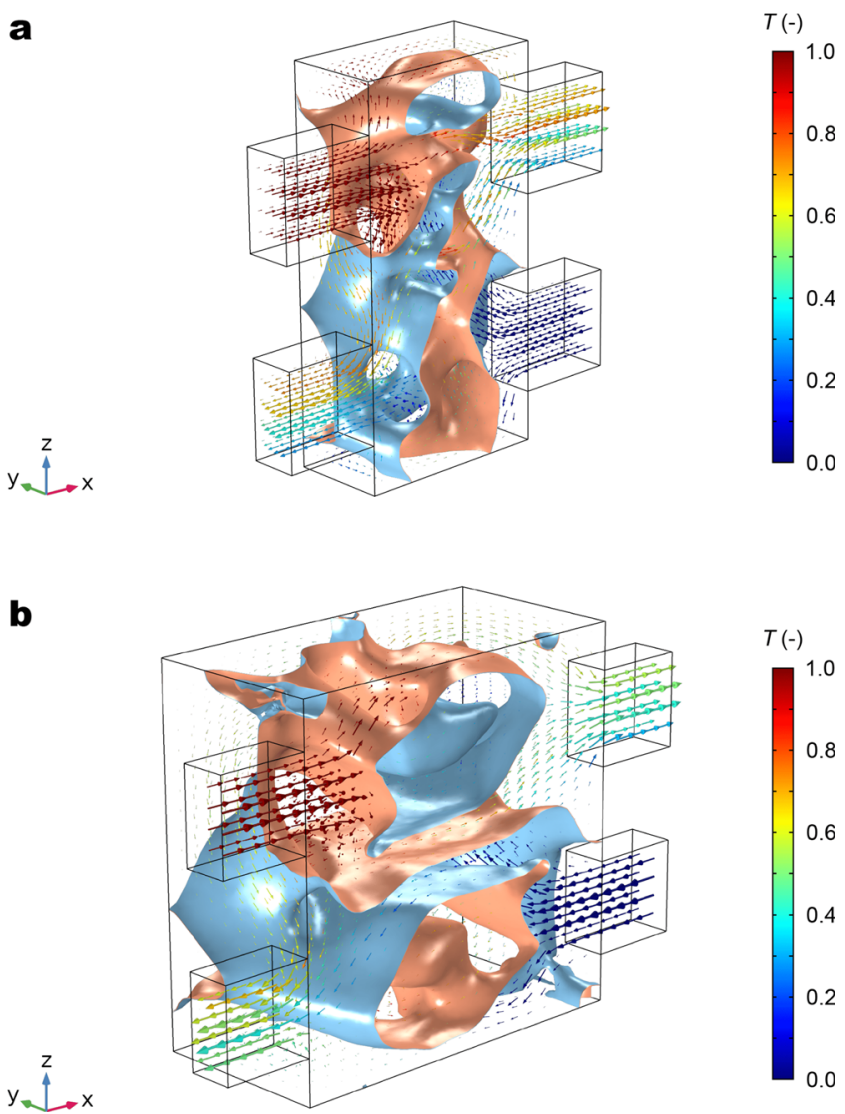

Fig. 17 Optimized result for different design domain size: a $L_{x}=2 L$, $L_{y}=2 L, L_{z}=4 L ; \mathbf{b} L_{x}=4 L, L_{y}=4 L, L_{z}=4 L$. Objective function values: a $J=0.213$; $\mathbf{b} J=0.433$ eased pressure loss limit. In contrast, increasing the Prandtl number corresponds with the strict requirement for total heat transfer. This can be explained by the fact that Péclet numbers of both cases in Figs. $14 \mathrm{~b}$ and 16 are identical; $P e_{1}=1400, P e_{2}=1400$. In addition, this similarity can be explained in the same way as the tendency of the temperature change of the fluids through the inlet to the outlet. That is, a higher $R e$ prevents any decrease in the temperature, due to a higher flow rate. This feature is also observed in a higher $\operatorname{Pr}$ due to a smaller heat conductivity.

\subsubsection{Effect of design domain size}

To investigate the effect of the design domain size, the optimization is performed in various $L_{x}, L_{y}$, and $L_{z}$. Figure 17 shows the optimized results for $L_{x}=2 L, L_{y}=$ $2 L$, and $L_{z}=4 L$, as well as for $L_{x}=4 L, L_{y}=4 L$, and $L_{z}=4 L$. The parameters are similar to the case of $L_{x}=4 L, L_{y}=2 L$, and $L_{z}=4 L$ presented in Section 4.3.

Although the flow channels are placed alternately regardless of the design domain size, different features are obtained in each case. For Fig. 17a, the branched channels are in the $z$ direction instead of the $x$ direction as found in the cases of Figs. 9 and 17b. Since the length in $x$ direction is short and the heat exchange domain is small, a relatively sharp bend is obtained. From Fig. 17b, thick and flat flow channels are realized when the length in $y$ direction is large. This is because the narrow flow channels are not necessary in case of a large heat exchange domain.
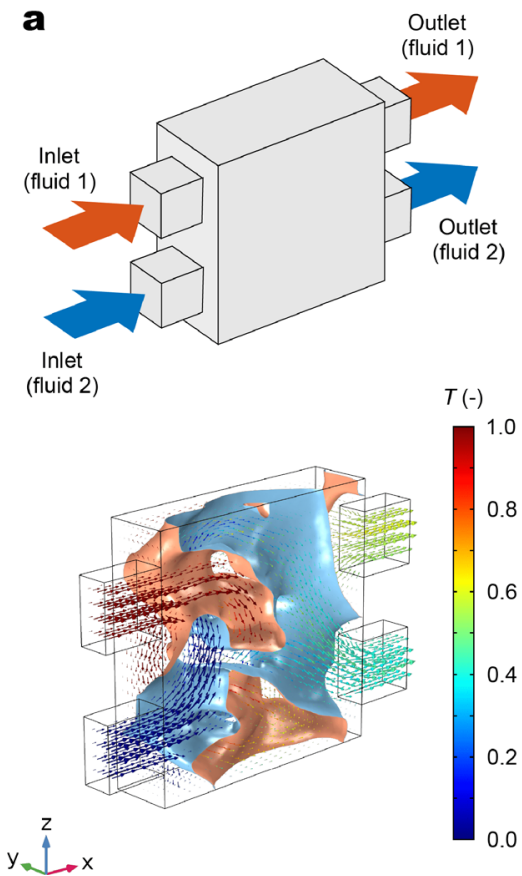
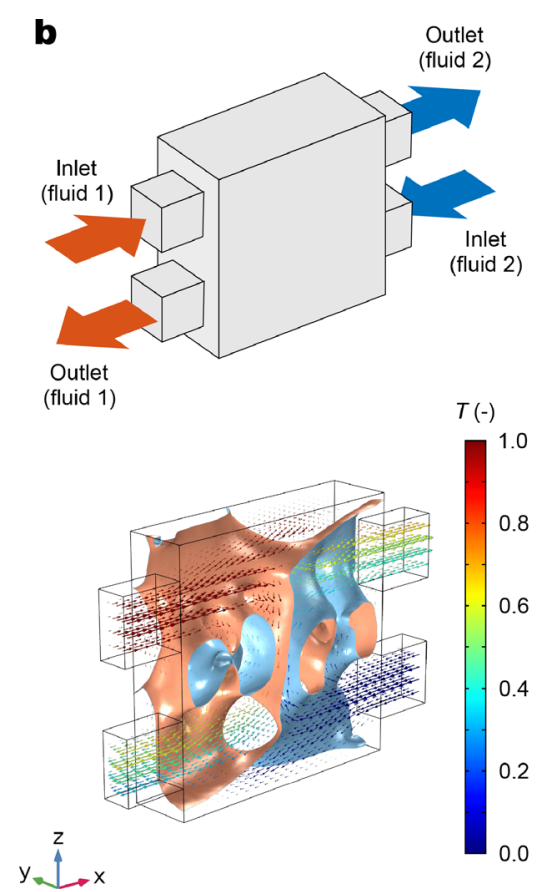

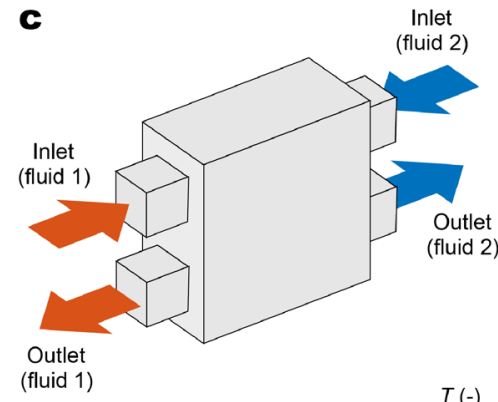

(fluid 1)

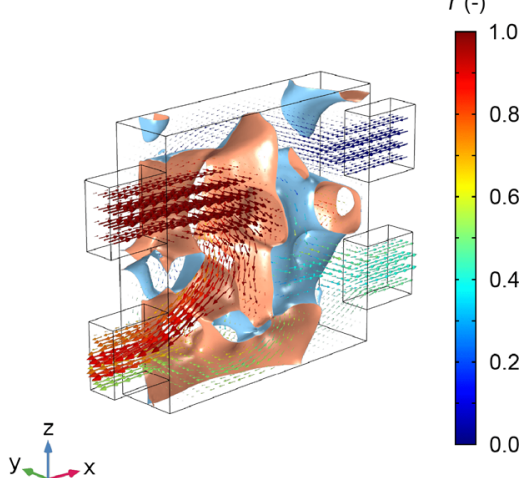

Fig. 18 Schematic diagram and optimized result of several flow arrangements: a parallel flow; b U-counter flow; c U-parallel flow. Objective function values: a $J=0.289$; b $J=0.294$; c $J=0.263$ 


\subsubsection{Effect of flow arrangement}

Finally, the effect of the flow arrangement, i.e., the arrangement of the inlet and the outlet in each fluid, is investigated. Figure 18 displays the schematic diagram and the optimized results. We denote the arrangement of Fig. 18b and c by "U-counter" and "U-parallel," respectively. The aim of this numerical example is to demonstrate that the proposed method can generate appropriate optimized configurations for each flow arrangement, whereas it is widely known that counter flow arrangements, as shown in Fig. 5, are generally more effective than other arrangements.

From Fig. 18a, the flow channels of each fluid are placed alternately at right angles in parallel flow. It is discovered that the heat is mainly exchanged in the upstream region, and the temperature is nearly unchanged in the downstream region. This is because both fluids have approximately the same temperature after passing the branched section, and downstream fluid 1 cannot be adjacent to the upstream fluid 2, and vice versa, unlike in the case of counter flow. Similar features are found in Fig. 18c, U-parallel flow. In Fig. 18b, the high temperature difference can be maintained in the whole heat exchange surface, which is similar to counter flow. Based on these results, it can be confirmed that the outlet of the fluid 1 should be close to the inlet of the fluid 2 , and vice versa, for efficient heat exchange.

\section{Conclusions}

In this paper, we proposed a density-based topology optimization method for two-fluid heat exchange problems. The novel aspect of the paper is that a representation model involving three states (two types of fluids and a solid wall) is defined using a single design variable field. To this end, we introduced two types of fictitious force terms, one in each flow field so that, essentially, the two fluids cannot mix.

We formulated the optimization problem as a maximization problem of the heat transfer rate under the fixed pressure loss. Based on the formulation, we investigated the effectiveness of the proposed method through threedimensional numerical examples.

In the numerical examples, we first conducted the verification of the proposed representation model in a simple analysis model that has a flat plate between fluid 1 and fluid 2. We then demonstrated the performance of an optimized design in comparison with a reference design. We demonstrated the dependency of the optimized design with respect to the design conditions, i.e., Reynolds number, Prandtl number, design domain size, and flow arrangements. Accordingly, it was found that the proposed method can generate effective optimized designs for optimization problem concerning the two-fluid heat exchange system.
Conversely, one aspect of the proposed representation model has an issue, namely, control of the solid wall thickness is difficult due to the use of the simple model for representing the three states. This is an unresolved issue, which we plan to study in future work. In addition, we hope to apply the proposed method to practical designs of heat exchangers (Webb and Kim 2005).

In the review process of this paper after the first original submission, we identified a related reference (Høghøj et al. 2020), which was recently uploaded to arxiv.org as a preprint. This reference also used a single design variable field for designing two-fluid heat exchangers via topology optimization, and provided three-dimensional numerical examples. Including this reference, as research focusing on topology optimization for two-fluid heat exchange is a developing field, as mentioned in Section 1, future work would need to comprehensively investigate the effectiveness of each method under the same design conditions.

Acknowledgments This work was partially supported by JSPS KAKENHI Grant Number 18K13674.

\section{Compliance with ethical standards}

Conflict of interest The authors declare that they have no conflict of interest.

Replication of results The necessary information for replication of the results is presented in this paper. The interested reader may contact the corresponding author for further implementation details.

Open Access This article is licensed under a Creative Commons Attribution 4.0 International License, which permits use, sharing, adaptation, distribution and reproduction in any medium or format, as long as you give appropriate credit to the original author(s) and the source, provide a link to the Creative Commons licence, and indicate if changes were made. The images or other third party material in this article are included in the article's Creative Commons licence, unless indicated otherwise in a credit line to the material. If material is not included in the article's Creative Commons licence and your intended use is not permitted by statutory regulation or exceeds the permitted use, you will need to obtain permission directly from the copyright holder. To view a copy of this licence, visit http:// creativecommonshorg/licenses/by/4.0/.

\section{References}

Alexandersen J, Aage N, Andreasen CS, Sigmund O (2014) Topology optimisation for natural convection problems. Int $\mathrm{J}$ Numer Methods Fluids 76(10):699-721

Alexandersen J, Sigmund O, Aage N (2016) Large scale threedimensional topology optimisation of heat sinks cooled by natural convection. Int J Heat Mass Transf 100:876-891

Alexandersen J, Andreasen CS (2020) A review of topology optimisation for fluid-based problems. Fluids 5(1):29

Andreasen CS, Gersborg AR, Sigmund O (2009) Topology optimization of microfluidic mixers. Int $\mathbf{J}$ Numer Methods Fluids 61(5):498-513 
Asmussen J, Alexandersen J, Sigmund O, Andreasen CS (2019) A "poor man's" approach to topology optimization of natural convection problems. Struct Multidiscip Optim 59(4):1105-1124

Bendsøe MP, Kikuchi N (1988) Generating optimal topologies in structural design using a homogenization method. Comput Methods Appl Mech Eng 71(2):197-224

Bendsøe MP, Sigmund O (2003) Topology optimization: theory, methods and applications. Springer, London

Borrvall T, Petersson J (2003) Topology optimization of fluids in Stokes flow. Int J Numer Methods Fluids 41(1):77-107

Chen CH, Yaji K, Yamasaki S, Tsushima S, Fujita K (2019) Computational design of flow fields for vanadium redox flow batteries via topology optimization. J Energy Storage 26:100990

Clausen A, Aage N, Sigmund O (2015) Topology optimization of coated structures and material interface problems. Comput Methods Appl Mech Eng 290:524-541

Coffin P, Maute K (2016) A level-set method for steady-state and transient natural convection problems. Struct Multidiscip Optim 53(5):1047-1067

Deaton JD, Grandhi RV (2014) A survey of structural and multidisciplinary continuum topology optimization: post 2000 . Struct Multidiscip Optim 49(1):1-38

Deng Y, Zhou T, Liu Z, Wu Y, Qian S, Korvink JG (2018) Topology optimization of electrode patterns for electroosmotic micromixer. Int J Heat Mass Transf 126:1299-1315

Dilgen SB, Dilgen CB, Fuhrman DR, Sigmund O, Lazarov BS (2018) Density based topology optimization of turbulent flow heat transfer systems. Struct Multidiscip Optim 57(5):1905-1918

Gersborg-Hansen A, Sigmund O, Haber RB (2005) Topology optimization of channel flow problems. Struct Multidiscip Optim 30(3):181-192

Guo K, Zhang N, Smith R (2018) Design optimisation of multi-stream plate fin heat exchangers with multiple fin types. Appl Therm Eng 131:30-40

Haertel JH, Nellis GF (2017) A fully developed flow thermofluid model for topology optimization of 3D-printed air-cooled heat exchangers. Appl Therm Eng 119:10-24

Haertel JH (2018) Design of thermal systems using topology optimization. PhD thesis, Technical University of Denmark

Hilbert R, Janiga G, Baron R, Thévenin D (2006) Multi-objective shape optimization of a heat exchanger using parallel genetic algorithms. Int J Heat Mass Transf 49(15-16):2567-2577

Høghøj LC, Nørhave DR, Alexandersen J, Sigmund O, Andreasen CS (2020) Topology optimization of two fluid heat exchangers. arXiv:200701759

Kambampati S, Kim HA (2020) Level set topology optimization of cooling channels using the Darcy flow model. Struct Multidiscip Optim 61:1345-1361

Kanaris A, Mouza A, Paras S (2009) Optimal design of a plate heat exchanger with undulated surfaces. Int J Therm Sci 48(6):11841195

Kawamoto A, Matsumori T, Yamasaki S, Nomura T, Kondoh T, Nishiwaki S (2011) Heaviside projection based topology optimization by a PDE-filtered scalar function. Struct Multidiscip Optim 44(1):19-24

Kobayashi H, Yaji K, Yamasaki S, Fujita K (2019) Freeform winglet design of fin-and-tube heat exchangers guided by topology optimization. Appl Therm Eng 161:114020

Koga AA, Lopes ECC, Nova HFV, De Lima CR, Silva ECN (2013) Development of heat sink device by using topology optimization. Int J Heat Mass Transf 64:759-772
Lazarov BS, Sigmund O (2011) Filters in topology optimization based on Helmholtz-type differential equations. Int J Numer Methods Eng 86(6):765-781

Lin S, Zhao L, Guest JK, Weihs TP, Liu Z (2015) Topology optimization of fixed-geometry fluid diodes. J Mech Des 137(8)

Matsumori T, Kondoh T, Kawamoto A, Nomura T (2013) Topology optimization for fluid-thermal interaction problems under constant input power. Struct Multidiscip Optim 47(4):571-581

Okkels F, Bruus H (2007) Scaling behavior of optimally structured catalytic microfluidic reactors. Phys Rev E 75(1):016301

Olesen LH, Okkels F, Bruus H (2006) A high-level programminglanguage implementation of topology optimization applied to steady-state Navier-Stokes flow. Int J Numer Methods Eng 65(7):975-1001

Papazoglou P (2015) Topology optimization of heat exchangers. Master's thesis, Delft University of Technology

Pollini N, Sigmund O, Andreasen CS, Alexandersen J (2020) A "poor man's" approach for high-resolution three-dimensional topology design for natural convection problems. Adv Eng Softw 140:102736

Sato Y, Yaji K, Izui K, Yamada T, Nishiwaki S (2017) Topology optimization of a no-moving-part valve incorporating Pareto frontier exploration. Struct Multidiscip Optim 56(4):839-851

Saviers KR, Ranjan R, Mahmoudi R (2019) Design and validation of topology optimized heat exchangers. In: AIAA Scitech 2019 forum, pp 1465

Scheithauer U, Schwarzer E, Moritz T, Michaelis A (2018) Additive manufacturing of ceramic heat exchanger: opportunities and limits of the lithography-based ceramic manufacturing (LCM). J Mater Eng Perform 27(1):14-20

Shah RK, Sekulic DP (2003) Fundamentals of heat exchanger design. Wiley, New York

Sigmund O, Maute K (2013) Topology optimization approaches. Struct Multidiscip Optim 48(6):1031-1055

Tawk R, Ghannam B, Nemer M (2019) Topology optimization of heat and mass transfer problems in two fluids-one solid domains. Numer Heat Transf Part B: Fund 76(3):130-151

Wang F, Lazarov BS, Sigmund O (2011) On projection methods, convergence and robust formulations in topology optimization. Struct Multidiscip Optim 43(6):767-784

Webb RL, Kim N (2005) Principles of enhanced heat transfer. NY, Taylor and Francis

Yaji K, Yamada T, Kubo S, Izui K, Nishiwaki S (2015) A topology optimization method for a coupled thermal-fluid problem using level set boundary expressions. Int J Heat Mass Transf 81:878888

Yaji K, Ogino M, Chen C, Fujita K (2018a) Large-scale topology optimization incorporating local-in-time adjoint-based method for unsteady thermal-fluid problem. Struct Multidiscip Optim 58(2):817-822

Yaji K, Yamasaki S, Tsushima S, Suzuki T, Fujita K (2018b) Topology optimization for the design of flow fields in a redox flow battery. Struct Multidiscip Optim 57(2):535-546

Yaji K, Yamasaki S, Fujita K (2020) Multifidelity design guided by topology optimization. Struct Multidiscip Optim 61(3):1071-1085

Zhao X, Zhou M, Sigmund O, Andreasen CS (2018) A “poor man's approach" to topology optimization of cooling channels based on a Darcy flow model. Int J Heat Mass Transf 116:1108-1123

Publisher's note Springer Nature remains neutral with regard to jurisdictional claims in published maps and institutional affiliations. 